\title{
INTERMEDIATE VALUE THEOREMS AND FIXED POINT THEOREMS FOR SEMI-CONTINUOUS FUNCTIONS IN PRODUCT SPACES
}

\author{
JEAN GUILLERME
}

(Communicated by Palle E. T. Jorgensen)

\begin{abstract}
We prove an intermediate value theorem for noncontinuous functions; as consequences, we obtain coincidence and fixed points theorems for nonmonotone and noncontinuous functions defined and with values in a product space $\mathbb{R}^{I}$. Some of them, even when the index set $I$ is a singleton, improve recent statements of S. Schmitd.
\end{abstract}

Let us consider the two following classical properties: a continuous or a nondecreasing function from $[0,1]$ into itself has a fixed point. Are these results related together? Another formulation of this question may be: is there a relation between the continuity and the monotonicity of a function? Of course the answer is yes, since we are working on $[0,1]$. More precisely, a nondecreasing function on $[0,1]$ is upper semi-continuous on the left and lower semi-continuous on the right. It is the semi-continuity properties which allow us to give intermediate value theorems, coincidence theorems and fixed point theorems.

We consider in this short paper not only real functions of a real variable but, more generally, functions defined and with values in a product space, $\mathbb{R}^{I}$; mostly, the assumptions on each coordinate function are right and/or left semicontinuities and a monotonicity condition which quasi-monotone functions possess; so we obtain in particular a generalization of a fixed point theorem of $S$. Schmitd [2] which has motivated our study.

Let $I$ be an arbitrary index set. An element $x:=\left(x_{i}\right)_{i \in I}$ of $\mathbb{R}^{I}$ will be also denoted by $x:=\left(x_{i}, x^{i}\right)$ where $x^{i}$ belongs to the product space $\mathbb{R}^{I \backslash\{i\}}$. Of course, the space $\mathbb{R}^{I}$ is equipped with the product order and so, for $x$ and $y$ in $\mathbb{R}^{I}$, we write $x \leq y$ if and only if $x_{i} \leq y_{i}$ for every $i$ in $I ;[x, y]$ is the set of points $z$ in $\mathbb{R}^{I}$ with $x \leq z \leq y$. In the sequel, two points $u$ and $v$ are fixed, with $u \leq v$.

Theorem 1 (Intermediate value theorem). Let $h:=\left(h_{i}\right)_{i \in I}$ be a function from $[u, v]$ in $\mathbb{R}^{I}$. Suppose that, for each $i$ in $I$ and each $x$ in $[u, v]$, the following properties are fulfilled:

(1.1) the function $h_{i}\left(\cdot, x^{i}\right)$ is lower semi-continuous on the right on $\left[u_{i}, v_{i}\right]$;

Received by the editors July 22, 1993 and, in revised form, October 22, 1993.

1991 Mathematics Subject Classification. Primary 47H10, 54H25; Secondary 26A15. 
(1.2) the function $h_{i}\left(\cdot, x^{i}\right)$ is upper semi-continuous on the left on $\left[u_{i}, v_{i}\right]$;

(1.3) the function $h_{i}\left(x^{i}, \cdot\right)$ is nondecreasing on $\left[u^{i}, v^{i}\right]:=\prod_{j \in I \backslash\{i\}}\left[u_{j}, v_{j}\right]$. Then, the interval $[h(v), h(u)]$ is contained in the set $h([u, v])$.

Proof. We suppose $h(v) \leq h(u)$, else there is nothing to prove. Let $\lambda$ be an element of $\mathbb{R}^{I}$ such that $h(v) \leq \lambda \leq h(u)$; we have to prove that the function $h$ takes the value $\lambda$ at a point of $[u, v]$. Let us set:

$$
H_{\lambda}:=\{x \in[u, v] \mid \lambda \leq(x)\}
$$

and for each $i$

$$
H_{\lambda, i}:=\left\{x_{i} \in\left[u_{i}, v_{i}\right] \mid \exists x^{i} \in\left[u^{i}, v^{i}\right] \lambda \leq h\left(x_{i}, x^{i}\right)\right\} .
$$

From the choice of $\lambda$, we remark that $u$ belongs to $H_{\lambda}$ and hence it makes sense to define the point $\bar{x}$ to be the least upper bound of $H_{\lambda} \quad\left(\bar{x}=\sup H_{\lambda}\right)$; we are going to show that $\lambda=h(\bar{x})$. We remark also that $u_{i}$ belongs to $H_{\lambda, i}$ and it is easy to verify, directly from the definitions, that $\bar{x}_{i}=\sup H_{\lambda, i}$.

In order to prove $\lambda \leq h(\bar{x})$, let us choose arbitrarily an index $i$ in $I$ and a real number $\mu, \mu>h_{i}(\bar{x})$. From (1.2), we find a neighborhood $U_{i}$ of $\bar{x}_{i}$ in $\left[u_{i}, v_{i}\right]$ such that $h_{i}\left(x_{i}, \bar{x}^{i}\right)<\mu$ for every $x_{i}$ in $U_{i}, x_{i} \leq \bar{x}_{i}$. Since $\bar{x}_{i}=\sup H_{\lambda, i}$, we can find a point $y_{i}$ in $H_{\lambda, i} \cap U_{i}$, with $y_{i} \leq \bar{x}_{i}$ (and hence $\left.h_{i}\left(y_{i}, \bar{x}^{i}\right)<\mu\right)$ and since $y_{i}$ belongs to $H_{\lambda, i}$ there exists a point $y^{i}$ in $\left[u^{i}, v^{i}\right]$ such that $\lambda \leq h\left(y_{i}, y^{i}\right)$, and in particular such that $\lambda_{i} \leq h_{i}\left(y_{i}, y^{i}\right)$. Then, the point $y:=\left(y_{i}, y^{i}\right)$ belongs to $H_{\lambda}$ and therefore $y \leq \bar{x}$ and in particular $y_{i} \leq \bar{x}_{i}$. Consequently, using the property (1.3), we obtain $\lambda_{i}<\mu$ and then $\lambda_{i} \leq h_{i}(\bar{x})$ from the arbitrary choice of $\mu$ and finally $\lambda \leq h(\bar{x})$ from the arbitrary choice of $i$.

We now want to show $h(\bar{x}) \leq \lambda$, that is, $h_{i}(\bar{x}) \leq \lambda_{i}$ for every $i$ in $I$, and so we fix arbitrarily an index $i$. If $\bar{x}_{i}=v_{i}$, then the result is clear by the property (1.3) and the choice of $\lambda\left(h_{i}(\bar{x})=h_{i}\left(\bar{x}_{i}, \bar{x}^{i}\right)=h_{i}\left(v_{i}, \bar{x}^{i}\right) \leq h_{i}\left(v_{i} v^{i}\right) \leq \lambda_{i}\right)$. Therefore we consider the case $\bar{x}_{i}<v_{i}$; let us suppose $\lambda_{i}<h_{i}(\bar{x})$. From (1.1), we find a neighborhood $V_{i}$ of $\bar{x}_{i}$ (in $\left[u_{i}, v_{i}\right]$ ) such that $\lambda_{i}<h_{i}\left(x_{i}, \bar{x}^{i}\right)$ for every $x_{i}$ in $V_{i}, x_{i} \geq \bar{x}_{i}$; let us take a point $y_{i}$ in $V_{i}, \bar{x}_{i}<y_{i}<v_{i}$, and let us consider the point $w:=\left(y_{i}, \bar{x}^{i}\right)$. Then we have $h_{i}(w) \geq \lambda_{i}$. Moreover we have also $w \geq \bar{x}$, which implies $w^{j} \geq \bar{x}^{j}$ for every $j$ in $I$, and in particular for $j \neq i$. For such $j$, we have $w_{j}=\bar{x}_{j}$ and we obtain, by $(1.3), h_{j}(w)=$ $h_{j}\left(w_{j}, w^{j}\right) \geq h_{j}\left(w_{j}, \bar{x}^{j}\right)=h_{j}\left(\bar{x}_{j}, \bar{x}^{j}\right)$ and so, from the first part of the proof $h_{j}(w) \geq \lambda_{j}$. Therefore we obtain $h(w) \geq \lambda$ and hence $w$ belongs to $H_{\lambda}$, which is in contradiction with the inequality $w_{i}>\bar{x}_{i}$. Thus, we have $h_{i}(\bar{x}) \leq \lambda_{i}$ and the proof is complete.

If we replace in Theorem 1 the function $h$ by the function $-h$, we obtain its dual form:

Corollary 2 (Intermediate value theorem). Let $h:=\left(h_{i}\right)_{i \in I}$ be a function from $[u, v]$ into $\mathbb{R}^{I}$. Suppose that, for each $i$ in $I$ and each $x$ in $[u, v]$, the following properties are fulfilled:

(2.1) the function $h_{i}\left(\cdot, x^{i}\right)$ is upper semi-continuous on the right on $\left[u_{i}, v_{i}\right]$;

(2.2) the function $h_{i}\left(\cdot, x^{i}\right)$ is lower semi-continuous on the left on $\left[u_{i}, v_{i}\right]$;

(2.3) the function $h_{i}\left(x_{i}, \cdot\right)$ is nonincreasing on $\left[u^{i}, v^{i}\right]$.

Then, the interval $[h(u), h(v)]$ is contained in the set $h([u, v])$. 
Important consequences of the preceding statements are the following coincidence or fixed point theorems.

Theorem 3 (Coincidence theorem). Let $f:=\left(f_{i}\right)_{i \in I}$ and $g:=\left(g_{i}\right)_{i \in I}$ be two functions from $[u, v]$ into $\mathbb{R}^{I}$. Suppose that, for each $i$ in $I$ and each $x$ in $[u, v]$, the following properties are fulfilled:

(3.1) the function $f_{i}\left(\cdot, x^{i}\right)$ is l.s.c. on the right and u.s.c. on the left on $\left[u_{i}, v_{i}\right]$;

(3.2) the function $g_{i}\left(\cdot, x^{i}\right)$ is u.s.c. on the right and l.s.c. on the left on $\left[u_{i}, v_{i}\right]$;

(3.3) the function $f_{i}\left(x_{i}, \cdot\right)$ is nondecreasing on $\left[u^{i}, v^{i}\right]$;

(3.4) the function $g_{i}\left(x_{i}, \cdot\right)$ is nonincreasing on $\left[u^{i}, v^{i}\right]$.

Suppose moreover that we have:

(3.5) $g(u) \leq f(u)$ and $f(v) \leq g(v)$.

Then, there exists a point $\bar{x}$ in $[u, v]$ such that $f(\bar{x})=g(\bar{x})$.

Theorem 4 (Fixed point theorem). Let $f:=\left(f_{i}\right)_{i \in I}$ be a function from $[u, v]$ into $\mathbb{R}^{I}$. Suppose that, for each $i$ in $I$ and each $x$ in $[u, v]$, the following properties are fulfilled:

(4.1) the function $f_{i}\left(\cdot, x^{i}\right)$ is l.s.c. on the right and u.s.c. on the left on $\left[u_{i}, v_{i}\right]$;

(4.2) the function $f_{i}\left(x_{i}, \cdot\right)$ is nondecreasing on $\left[u^{i}, v^{i}\right]$;

Suppose moreover that we have:

(4.3) $u \leq f(u)$ and $f(v) \leq v$.

Then, there exists a point $\bar{x}$ in $[u, v]$ such that $f(\bar{x})=\bar{x}$.

Theorem 5 (Fixed point theorem). Let $g:=\left(g_{i}\right)_{i \in I}$ be a function from $[u, v]$ into $\mathbb{R}^{I}$. Suppose that, for each $i$ in $I$ and each $x$ in $[u, v]$, the following properties are fulfilled:

(5.1) the function $g_{i}\left(\cdot, x^{i}\right)$ is u.s.c. on the right and l.s.c. on the left on $\left[u_{i}, v_{i}\right]$;

(5.2) the function $g_{i}\left(x_{i}, \cdot\right)$ is nonincreasing on $\left[u^{i}, v^{i}\right]$.

Suppose moreover that we have:

(5.3) $g(u) \leq u$ and $v \leq g(v)$.

Then, there exists a point $\bar{x}$ in $[u, v]$ such that $g(\bar{x})=\bar{x}$.

Proof. In order to obtain Theorem 3, it is sufficient to apply Theorem 1 with $h:=f-g$ (and at the intermediate value $\lambda=0$ ). To obtain Theorem 4 (resp. 5 ), it suffices to apply Theorem 3 with the identity in place of $g$ (resp. $f$ ). (For example, if $g(x)=x$, then $g_{i}\left(x_{i}, \cdot\right)=x_{i}$; this function is constant, and hence it is nonincreasing.)

Remarks. (6.1) Tarski Theorem [3], applied to functions defined on a product of intervals, states that a nondecreasing function $f:=\left(f_{i}\right)_{i \in I}$ from $[u, v]$ into itself has a fixed point. This result is contained in Theorem 4, since for every $i$ in $I$ and every $x=\left(x_{i}, x^{i}\right)$, the function $f_{i}\left(x_{i}, \cdot\right)$ is then nondecreasing, and also the function $f_{i}\left(\cdot, x^{i}\right)$ is nondecreasing, and hence it is 1.s.c. on the right and u.s.c. on the left on $\left[u_{i}, v_{i}\right]$, as we have already noticed.

(6.2) Suppose that $w$ is a real function of a real variable. It is easy to verify that, for every $x$, the following implication holds:

$$
\lim \inf _{t \rightarrow 0, t>0} \frac{w(x+t)-w(x)}{t}>-\infty \Rightarrow \lim \inf _{t \rightarrow 0, t>0} w(x+t) \geq w(x) .
$$


Of course, similar implications related to the lower limit on the left and upper limits on the right or on the left also hold, but not the inverses. Consequently, Theorems 4 and 5 improve strictly S. Schmitd's results [2].

We recall here that the monotonicity condition (hypothesis (1.3) for instance) is weaker than quasimonotonicity (see also [2] for the definition), and hence that our results apply to this class of functions.

(6.3) The proof shows that, in Theorem 4, the point $\bar{x}$ is the greatest fixed point of $f$; if we had defined, in Theorem 1 , the point $\underline{x}$ to be the greatest lower bound of the set $K_{\lambda}:=\{x \in[u, v] / h(x) \leq \lambda\}$, then we would obtain, in Theorem 4, that the point $\underline{x}$ is the smallest fixed point of $f$. Similar remarks can also be made for Theorem 5 .

(6.4) In [1], Hu recalled the notions of upper-right limit, lower-right limit, and so on, of a function $f$, but he does not really use these notions; they lead however directly to the notions of upper semi-continuity on the right, lower semi-continuity on the right, and so on, of the function $f$, notions which are the key of the present results!

To conclude, we underline that we have obtained here not only fixed point theorems but also intermediate value theorems and coincidence theorems for functions defined and with values in $\mathbb{R}^{I}$, where the index set $I$ is not necessarily denumerable. Moreover, and in the opposite direction, the preceding theorems hold of course also when the set $I$ is a singleton; in this case, the statements are particularly simple; for example, Theorem 5 becomes:

Suppose that the real function $k$ is u.s.c. on the right and l.s.c. on the left on a real interval $[a, b] ;$ if $k(a) \leq a$ and $b \leq k(b)$, then the function $k$ has a fixed point in $[a, b]$.

\section{REFERENCES}

1. S. Hu, Fixed points for discontinuous quasi-monotone maps in $\mathbb{R}^{n}$, Proc. Amer. Math. Soc. 104 (1988), 1111-1114.

2. S. Schmitd, Fixed points for discontinuous quasi-monotone maps is sequence spaces, Proc. Amer. Math. Soc. 115 (1992), 361-363.

3. A. Tarski, A lattice-theoretical fixpoint theorem and its applications, Pacific J. Math. 5 (1955), 285-309.

Départment de Mathematiques, U.R.A. no1586, 123 Avenue A. Thomas, 87060 Limoges Cedex France

E-mail address: guillerm@cict.fr 